\title{
Kinematics Simulation of Planar 3-DOF Parallel Mechanism
}

\author{
Chao Jia ${ }^{*}, 1$, Allam Maalla ${ }^{1}$, Fanshu Kong ${ }^{2}$ and Xijuan Guo ${ }^{2}$ \\ ${ }^{1}$ Guangzhou College of Commerce, School of Information Technology and Engineering, Guangzhou College of Commerce \\ Guangzhou, China, 511363 \\ ${ }^{2}$ Yanshan University, School of Information Technology Qinhuangdao 066004
}

\begin{abstract}
First, this paper establishes a three-dimension simulation model of the planar 3-dof parallel mechanism, and realized its kinematics simulation; Second, the velocity and acceleration curves of the mechanism are simulated by differential method and influence coefficient method, and the Singular configuration point of the mechanism are confirmed; Finally, analyzing the Singular configuration point of the mechanism, and obtained The influence of mechanism size change on kinematics performance.
\end{abstract}

Keyword-planar 3-dof parallel mechanism; kinematics simulation; Singular configuration point; kinematics performance

\section{INTRODUCTION}

As the development of parallel mechanism technology, small degrees of freedom parallel mechanism is becoming more and more popular because of its simple structure, economy and broad prospect of applying. At present, its unique structure renders the study aiming at its performance indices very limited, The understanding of kinematics and dynamics of the parallel mechanism is not sufficient ${ }^{[1][2][3]}$. In recent years, Liu Xinjun etc. ${ }^{[4][5]}$ have studied the velocity performance index of spherical planar two or three degrees of freedom parallel mechanism; Gao feng etc. ${ }^{[6][7][8]}$ have studied acceleration performance index of small degrees of freedom parallel mechanism; Huang zhen etc $^{[9][10]}$ makes use of fictitious mechanism influence coefficient method discuss theoretically the velocity and acceleration performance indices, But the actual researches need to go deep into. zhang xuetao etc. ${ }^{[11][12]}$ carries out The development and experimental research of the planar parallel robot experiment system .In view of this, the study of the physical model and kinematics performance of the planar 3dof parallel mechanism is of great practical value .This paper utilizes differential coefficient and influence coefficient methods renders simulation curves on mechanism velocity and acceleration , and analyzes the Singular configuration point of the mechanism and the influence of mechanism size change on kinematics performance .

\section{I. PLANAR 3-DOF PARALLEL MECHANISM MODEL}

The Planar 3-dof parallel mechanism (shown in figure 1) is a symmetric mechanism , and each branch chain's kinematic pair is basic pair .It is composed of three driving rods ,three passive rods and one triangular working platform .Among them ,there is revolute pair between each rod ,namely $A, G, B, H, C, I$ are all revolute pair .There is spherical pair between each rod and the triangular working platform ,namely D,E, F are all spherical pair. Three driving rods $\mathrm{AG}, \mathrm{BH}, \mathrm{CI}$ and three passive rods $\mathrm{GD}, \mathrm{HE}, \mathrm{IF}$ belong to controlling mechanism . Taking the initial Angle and rotation amplitude of the three active rods as input parameters, it can control mechanism to make different motion by adjusting input parameters' values and starting values.

The working platform DBF is the output of the mechanism, which can be installed on the working platform with various devices to enable the mechanism to accomplish different functions according to different requisition.

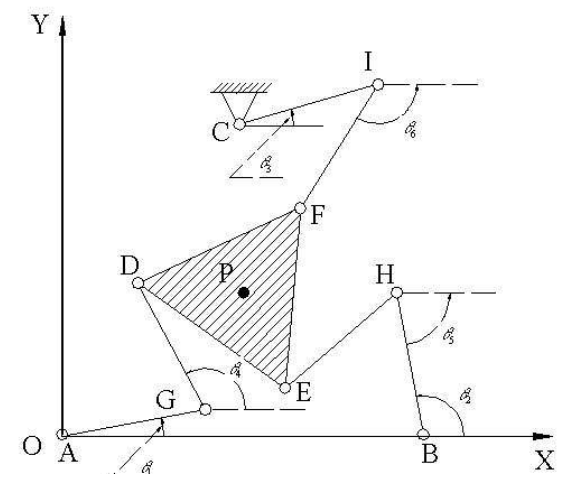

FIGURE I. MECHANISM 3-DOF SKETCH

\section{ESTABLISHMENT OF ENTITY SIMULATION MODEL}

First, the physical shape of each component is constructed, then the graphic attribute is set, and the location of each part is located. The simulation of each component takes a different approach. Fig. 2 shows the simulation model of the mechanism.

\section{A. Six rods}

AG, GD, BH, HE, CI, IF six rods, the simulation model method is the same, Take AG rod for example.

Taking $\mathrm{A}$ and $\mathrm{G}$ two points as the center of the two ends of the rod, using $A, G$ as the base point and the expansion 
value ${ }^{q}$ as reference to extend to eight points on the four sides, as the eight vertices of the cuboid.

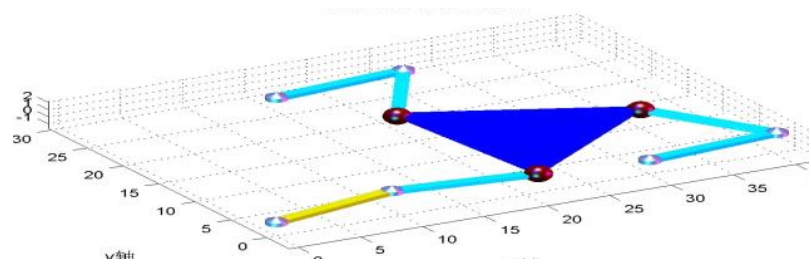

FIGURE II. ENTITY SIMULATION MODEL OF THE MECHANISM

Use eight vertices to fill three $4 \times 6$ matrices defining the $X, Y, Z$ coordinate values of cube's each surface. Among them, 4--each surface is determined by four vertices, $6--$ each cuboid is determined by six surfaces. Then, the algorithm of the automatic closed polygon is used to draw the polygon surface of the rod to form the stereogram, and the handle of the graph object is generated.

\section{B. Working platform}

Expand the work platform's three points (D, E, F) up to six points. The side matrixes of the work platform are first filled, consisting of three 4-by-3 matrices, 4--each side has four points, $3--$ totally three sides. Refill the upper and lower surface matrix, which consists of three 3-by-2 matrices, $3--$ each side has three points, $2--$ totally two sides . Finally, the automatic closed polygon algorithm is used to draw the three-dimensional drawing of the working platform, and the handle of each surface is also generated.

\section{Spherical pair}

Firstly, the coordinate matrix of the unit sphere is generated and the grid ball is drawn using the threedimensional grid surface generation algorithm, and create handle.

\section{Revolutepair}

First, the graph coordinate is generated and the model is generated by using the three-dimensional shadow surface rendering algorithm, and return handle at the same time.

\section{SimUlation ON MOTION PROCESS OF MECHANISM}

\section{A. Establishment of dynamic model}

Represent the movement of the mechanism, that is,the changes of the various components in the movement process, including the change of the shape of each part and the change of position.

The embodiment of shape changes mainly is the changes of shape of each component. Because to one view point, each component's different obliquity may produce different vision image. Therefore, we import obliquity as parameter when building 3-dimension matrices of each component's endpoints. When extending each point's X coordinate matrix, we use the expansion value ${ }^{q}$ times the positive spin of the Angle, and $\mathrm{Y}$ coordinate matrix uses $q$ times the residual value of the Angle, $\mathrm{Z}$ coordinate matrix keeps unchanged .We can attain more authentic dynamic effect of the model to do so.

The changes of position mainly depend on the dynamic changes of $\mathrm{A} \sim \mathrm{I}$. set the position attribute value of each component, and carry out The coordinate transformation of A I, to realize the dynamic change effect of the position of the mechanism.

The implementation of all the motion simulation discussed above is based on the coordinate transformation of $\mathrm{A} \sim \mathrm{I}$.

$$
\begin{aligned}
& A=[0,0] \quad B=\left[{ }^{L d s j}, 0\right] \\
& C=\left[L d s j / 2, \sqrt{3}_{*} L d s j / 2\right] \\
& G=\left[A(1)+L z l c * \cos \left({ }^{\theta_{1}}\right), A(2)+L z l c * \sin \left({ }^{\theta_{1}}\right)\right] \\
& H=\left[B(1)+L z l c * \cos \left({ }^{\theta_{2}}\right), B(2)+L z l c * \sin \left({ }^{\theta_{2}}\right)\right] \\
& I=\left[{ }^{C}(1)+L z l c * \cos \left({ }^{\theta_{3}}\right),{ }^{C}(2)+L z l c * \sin \left({ }^{\theta_{3}}\right)\right] \\
& D=\left[G_{(1)+} L z l c * \cos \left({ }^{\theta_{4}}\right), \mathrm{G}(2)+L z l c * \sin \left(\theta_{4}\right)\right] \\
& E=\left[H(1)+L z l c * \cos \left(\theta_{5}\right), \mathrm{H}(2)+L z l c * \sin \left(\theta_{5}\right)\right] \\
& F=\left[I(1)+L z l c * \cos \left(\theta_{6}\right), I(2)+L z l c * \sin \left({ }^{\theta_{6}}\right)\right] \\
& P=(D+E+F) / 3 \\
& L d s j \text { - side of triangle } A B C \\
& \text { Lzlc - length of driving rod } \\
& \text { Lzld - length of passive rod } \\
& \text { Lxsj_side of triangle } D E F
\end{aligned}
$$

When calculating the coordinates of D, E and F using the sides of the triangle $D E F$, the ${ }^{f \text { slove }}$ function is applied to solve the nonlinear equation. The setting of initial value is the key question. In the process of motion simulation, because the equation's solution is incessant change, if the initial value is set to a fixed value, not only affect the simulation speed, and because the equation is more solution, could mutate and cause system instability. The solving strategy is to set the initial value with position solution that was gotten at the previous moment.

\section{B. Constraint judgment in the process of motion}

The Planar 3-dof being a parallel mechanism, every rod is bound to contain each other, and the deformation of the mechanism can be changed beyond the elastic deformation limit. Therefore, the constraint on the freedom movement is: flexing of each side of the Working platform is less than given value; the length flexing of driving rods is less than given value; the length flexing of passive rods is less than given value. These set values are set according to the elastic deformation limit of the component. 
C. Motion trajectory simulation

Initial state: $L d s j=30, \quad L x s j=17, \quad$ Lzlc $=11$; $\theta 1=0^{\circ}, \theta 2=0^{\circ}, \theta 3=0^{\circ}, \Delta t=\pi / 160 ;$;angular velocity of three driving rods: $\varpi=(1,0.9,1) . \mathrm{t}=2.2259$, $\mathrm{t}=2.2455$, the simulation result illustrated as in Fig.3,4.

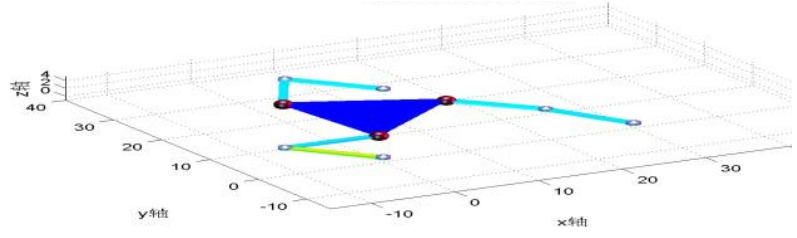

FIGURE III.MODEL POSTURE FOR TIME ${ }^{t}=2.2259$

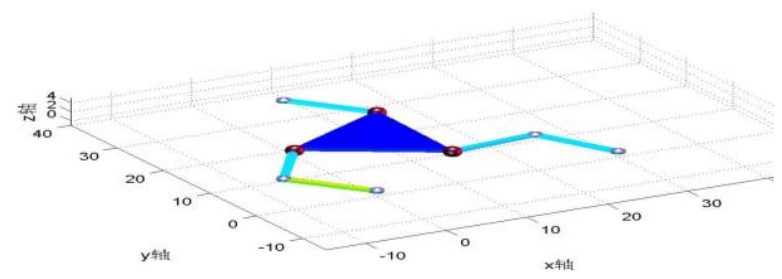

FIGURE IV. MODEL POSTURE FOR TIME $\mathrm{T}=2.2455$

The motion simulation of the mechanism model, in two consecutive times (from $\mathrm{t}=2.2259$ to $\mathrm{t}=2.2455$ ), has a mutation in its configuration (rods $\mathrm{BH}, \mathrm{EH}$ ). The singular position and posture is between $\theta 1=127.5347^{\circ}$. $\theta 1=128.6577^{\circ}$, This is the Singular configuration point of the mechanism. Velocity algorithm

\section{SimUlation ON Velocity AND ACCELERATION}

A. Velocity algorithm

1) First order comprehensive influence coefficient matrix method

The first order comprehensive influence coefficient matrix is $\left[G_{q}^{H}\right]$, The velocity of point $P$ is:

$\mathbf{v}=\left[G_{q}^{H}\right]_{*} \&$

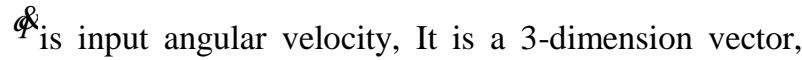
represents the input angular velocity of three driving rods; $\mathbf{v}$ is also a 3-dimension vector, represents the rotational angular velocity of the working platform, the linear velocity of $\mathrm{P}$ along $\mathrm{x}$-axis and along $\mathrm{y}$-axis .

2) Differential coefficient method

$P$ at the time of $t, v=(P(t 2)-P(t 1)) / \Delta t$

When $\Delta t$ is enough short, the velocity curve can be approximated.

B. Acceleration algorithm
1) second order comprehensive influence coefficient matrix method

The second order comprehensive influence coefficient matrix is $\left\lfloor H_{q}^{H}\right\rfloor$, The acceleration of point $P$ is:

$$
\left.a=\left[G_{q}^{H}\right]_{*} \&_{+} \mid H_{q}^{H}\right\rfloor_{*} \&
$$

$a$ is a 3-dimension vector, It represents the $P$ 's angular acceleration, the line acceleration along $\mathrm{x}$-axis and along $\mathrm{y}$ axis; ${ }^{\&}$ is input angular velocity, It is a 3 -dimension vector, represents the input angular velocity of three driving rods.

2) Differential coefficient method

$P$ at the time of $t, a=(\mathbf{v}(2)-\mathbf{v}(1)) / \Delta t$

When $\Delta t$ is enough short, the acceleration curve can be approximated.

C. Simulation analysis

Initialstate:

$\theta 1=0^{\circ}, \theta 2=0^{\circ}, \theta 3=0^{\circ}$,

$\&=(1,0.9,1), \theta=(0,0,0), \Delta t=\pi / 160$

The simulation diagram is shown in Fig. 5 - Fig. 11.

It can be seen from the figures: 1.The velocity and acceleration simulation curves drawn by two methods are in perfect agreement. 2. By changing the size of the mechanism, we get Fig.11(a) and Fig.11(b). Comparing two figures, the shorter the side of $\triangle A B C$ is, and the longer the side of $\triangle D E F$ is, the stronger the amplitude of the acceleration of the mechanism. This indicates that the stronger the inertia force of the mechanism, the worse the performance of the mechanism. 3. The simulation curves of mechanism velocity drawn by two methods reflect the singular configuration point of the mechanism, and the positions are all between $\theta 1=127.5347^{\circ}$ and $\theta 1=128.6577^{\circ}$. As shown in Figure 5a and Figure 6.

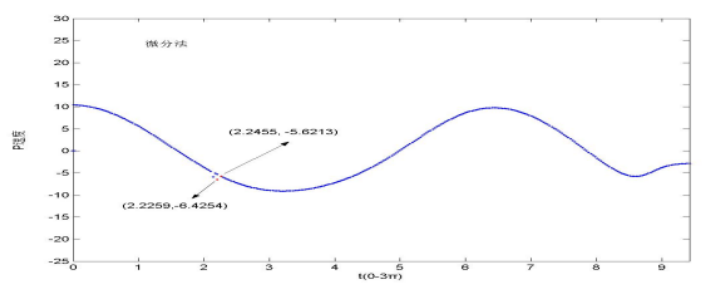

FIGURE V. (A) DIFFERENTIAL COEFFICIENT METHOD TO GET LINE SPEED OF P 


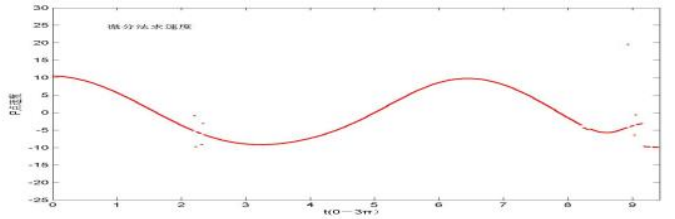

FIGURE V (B) DIFFERENTIAL COEFFICIENT METHOD TO GET LINE VELOCITY OF P(SIMULATION CONDITION IS SAME AS PICTURE 5A)

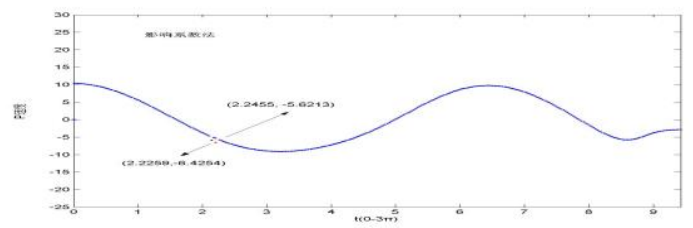

FIGURE VI. INFLUENCE COEFFICIENT METHOD TO GET LINE VELOCITY OF OUTPUT POINT P

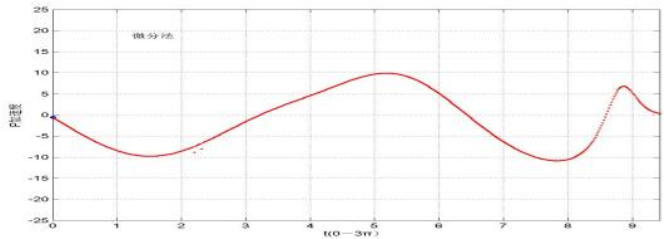

FIGURE VII. DIFFERENTIAL COEFFICIENT METHOD TO GET THE LINE VELOCITY OF P

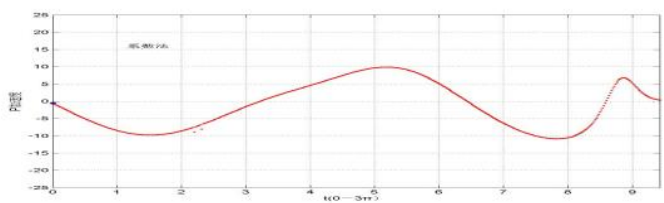

FIGURE VIII. INFLUENCE COEFFICIENT METHOD TO GET LINE ACCELERATION OF POINT P

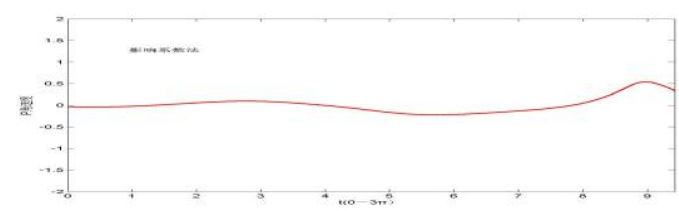

FIGURE IX. INFLUENCE COEFFICIENT METHOD TO GET THE ROTATIONAL ANGULAR VELOCITY OF WORKING PLATFORM

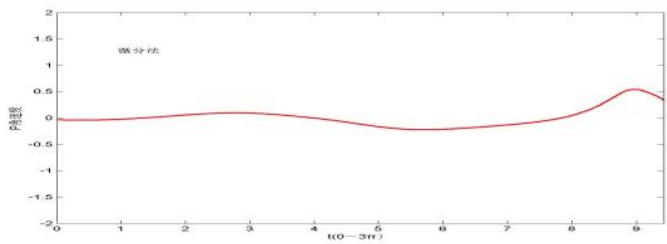

FIGURE X. DIFFERENTIAL COEFFICIENT METHOD TO GET THE ROTATIONAL ANGULAR VELOCITY OF WORKING PLATFORM

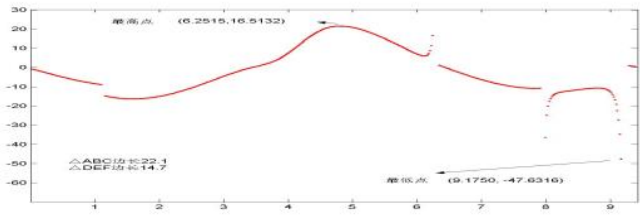

FIGURE XI (A) DIFFERENTIAL COEFFICIENT METHOD TO GET THE LINE VELOCITY OF MECHANISM

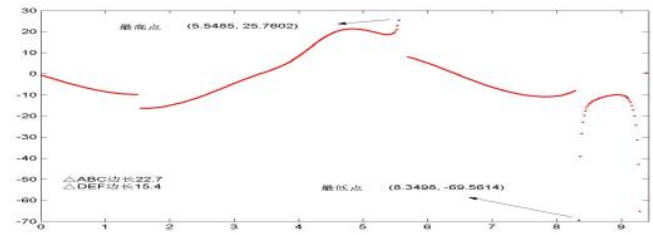

FIGURE XII (B) DIFFERENTIAL COEFFICIENT METHOD TO GET THE LINE ACCELERATION OF MECHANISM DYNAMIC MODELING AND ANALYSIS OF A PARALLEL MANIPULATOR USING SIMULINK AND SIMMECHANICS

\section{CONCLUSIONS}

In this paper, a 3-d entity simulation model of planar 3dof parallel mechanism and its kinematics simulation algorithm are established, and the position of the Singular configuration point of the mechanism is simulated. The motion velocity and acceleration curve of the mechanism are used to further confirm the Singular configuration point of the mechanism. The velocity and acceleration curves of the mechanism simulated by the two methods are in perfect agreement, which lays the foundation for further analysis of the planar parallel mechanism. By changing the size of the mechanism and comparing the acceleration curve, the influence of the size change of the mechanism on the kinematics performance of the mechanism is simulated.

\section{ACKNOWLEDGEMENT}

This research was financially supported by Characteristic Key Subject of E-commerce Construction Project of Guangzhou College of Commerce Foundation, project No. TSZDXK201601. And National Natural Science Research Project "Project Title: Construction and Application of Ecommerce Big Data Analysis Platform (Third Party), project No. (17BQNS01002).

\section{REFERENCES}

[1] AI Qing-lin, ZU Shun-jiang, XU Fang. Review of kinematics and singularity of parallel manipulator[J]. Journal of Zhejiang University (Engineering Science),2012,46(3):1345-1359.

[2] FENG Zhiyou, LI Yonggang, ZHANG Ce, et al. Present State and Perspectives of Research on Kinematics and Dynamics of Parallel Manipulators[J]. China Mechanical Engineering, 2006,17( 9) : 979984.

[3] LIU Xinjun, YU jingjun, WANG Guobiao, et al. Research trend and scientific challenge of robotics [J]. China science foundation, 2016,9(5):425-431.

[4] LIU Xinjun, WU Chao, WANG Jinsong, et al. Attitude description method of [PP]S type parallel robotic mechanisms[J]. Journal of mechanical engineering, 2008,44(10):19-23. 
[5] WANG Bo, LIU Xiang- dong, HAN Qiang, et al. Pose Analysis of 3DOF Planar Parallel Mechanism[J]. Machinery Manufacturing \& Automation, 2012(1):170-171+182.

[6] GAO Feng. Design Theory and Key Application Technology of Parallel Robots[J]. Journal of Hebei University of Technology, 2004,33( 2): 83-89.

[7] GAO Feng, GUO Weizhong. Thinking of the Development Strategy of Robots in China[J]. Journal of mechanical engineering, 2016,52(7):1-5.

[8] ZHOU Dongdong, FAN Jun. Error Analysis of 3- DOF Planar Parallel Robots[J]. Machine tool \& hydraulics,2017,45(3):6-9.

[9]HUANG Zhen, LIU Jingfang, ZENG Daxing. The general method of mechanism freedom analysis based on constraint spiral theory[J]. Science in China,2009, 1(20): 84-93.

[10] LIU Shanzeng, YU Yueqing, SI Guoning, et al. Kinematic and Dynamic Analysis of a Three-degree-of-freedom Parallel Manipulator[J]. Journal of mechanical engineering, 2009,45(8):11-17.

[11] ZHANG Xuetao, YANG Jianxin, YU Yueqing. High-speed flexible plane 3-rrr parallel robot experiment system development, Machine design \& research,2008, 5(11):39-41+46.

[12] WANG Yingbo, HUANG Qitao, ZHENG Shutao, et al. Dynamic modeling and analysis of a parallel manipulator using Simulink and SimMechanics[J]. Journal of Harbin Engineering University,2012,33(1):100-105. 